\title{
Integrin and Adhesion Regulation of Autophagy and Mitophagy
}

\author{
Eric A. Nollet and Cindy K. Miranti \\ Additional information is available at the end of the chapter \\ http://dx.doi.org/10.5772/55398
}

\section{Introduction}

Cell differentiation is a dynamic process that generates a functionally distinct cell from its progenitor. For example, human erythrocytes lack most organelles - including a nucleus while erythrocyte precursors have a complete set of organelles. Autophagy plays a critical role in organelle elimination in differentiating erythrocytes. On the other hand, most other differentiated cells in the body do not lose their organelles, and would not seem to heavily depend on autophagy during differentiation. However, evidence indicates that these cells require regulated autophagy during the differentiation process. For example, during keratinocyte differentiation a basal cell detaches from the basement membrane and is pushed to the upper strata, and as the cell ascends its intracellular contents are replaced with copious amounts of keratin. Drastic changes such these require cells to eliminate or turn over a large amount of biomass. Differentiation also causes a shift in signaling and survival pathways. An example of this is the prostate gland where the luminal cells require PI3K for survival while the undifferentiated basal cells do not; they depend on MAPK signaling for their survival. As we will discuss below, several different cell types use and require the autophagic pathway to properly differentiate and survive. While links between autophagy and differentiation are rapidly being identified, the mechanisms that trigger autophagic processes during differentiation are poorly understood. Genetics has served as a powerful tool for identifying the components of the autophagy machinery, but how they are integrated with cellular cues that trigger differentiation need further characterization. We will discuss the recently identified link between autophagy and cell adhesion, and its role in cellular differentiation and survival. 


\section{Role of autophagy during differentiation}

\subsection{Erythrocytes}

Erythrocytes are especially unique cells; they lack a nucleus, internal membrane-bound organelles, and ribosomes, and they are packed with the oxygen transporter hemoglobin. The cell arises from reticulocytes, a nucleated progenitor capable of generating the needed surplus hemoglobin and the erythrocyte itself [1,2]. Generating such a simple cell requires processes that eliminate organelles not essential for fully differentiated erythrocytes. A series of publications from 2008 to present not only elucidated the involvement of autophagy during reticulocyte differentiation, but also discovered that the mitochondria are specifically targeted for autophagy by a protein called Nix [3-7].

Nix and its related family member, Bnip3, are unique mitochondrial-localized BH3-only proteins. Although they can induce apoptosis when over expressed like most of the BH3-only proteins, Nix and Bnip3 function to stimulate autophagy and mitophagy as well. The mitophagic function of Nix was discovered in differentiating reticulocytes. Researchers knew that Nix expression dramatically increases in the terminal stage of reticulocyte differentiation, but the purpose for this remained unknown for several years [8]. In a relatively simple experiment, researchers harvested erythrocytes from $\mathrm{Nix}^{-/-}$mice and using mitotrophic dyes quantified mitochondria-containing erythrocytes. A significant population of the $\mathrm{Nix}^{-/}$erythrocytes still contained mitochondria. The absence of Nix did not affect LC3 levels, and autophagosomes were still present. However, the autophagosomes in $\mathrm{Nix}^{-/}$erythrocytes contained significantly less mitochondria than the wild type controls [4].

Erythrocytes from $\mathrm{Ulk}^{-}$mice retained mitochondria and ribosomes, indicating the necessity of the general autophagy program in reticulocyte differentiation. Inhibiting autophagy with the class III-PI3K inhibitor 3-methyladenine (3-MA) in wild type reticulocytes prevented mitochondrial clearance as well. The peripheral blood in $\mathrm{Ulk}^{-1-}$ mice contained an increased number of reticulocytes indicating a reduced capacity to be converted to erythrocytes [3]. Transplantation of fetal liver cells from Atg7 ${ }^{-}$mice into irradiated wild type mice resulted in overall fewer erythrocytes, and these erythrocytes contained mitochondria.[7]. Thus, both general macroautophagy, and organelle-specific autophagy is required for erythrocyte differentiation. The above studies focused on mitochondria, but whether specific autophagic targeting of other organelles, such as ribosomes and ER, is mediated by Nix or other organelle-specific factors during reticulocyte differentiation remains unanswered. Bnip3, like Nix, localizes to the ER, and researchers recently demonstrated that Bnip3 targets the ER for autophagy [9]. This raises the possibility that Nix may also target the ER for autophagy. Targeted autophagic degradation of ribosomes, termed ribophagy, occurs in S. cerevisiae. However, whether selective ribophagy occurs in higher eukaryotes remains unknown [10]. These questions would be interesting to answer in the reticulocyte differentiation model. 


\subsection{Lymphocytes}

In the same Atg $7^{--}$transplantation model, there was a four-fold reduction in the number of white blood cells and nine-fold reduction of lymphoid cells [7]. This observation was further validated in hematopoietic-specific stem, fetal, and adult cell Atg7 knockout mouse models [9, 10, 11]. Mitochondrial accumulation was observed in both $\mathrm{CD} 8^{+}$and $\mathrm{CD} 4^{+} \mathrm{T}$-cells, and these cells succumbed to apoptosis in vitro more readily than the wild type controls [11]. T-cell specific knockouts of Atg5 and Atg7 also displayed increased amounts of mitochondria and were more susceptible to apoptosis $[12,13]$. Considering that the overall population of mature $\mathrm{T}$ lymphocytes decreased and sensitivity to apoptosis increased in the autophagy-deficient $\mathrm{T}$ lymphocyte models, it seems that the lymphopenia occurs due to excess cell death. Puo et al tested this by stimulating T-cell proliferation in wild type and Atg $5^{-1-} \mathrm{T}$-cells and quantifying the amount of daughter cells produced by using the stable dye, 5,6-carboxyfluorescein diacetate succinimidyl ester (CFSE). CFSE diffuses into both cells during mitosis, causing a decrease in individual cell fluorescent intensity with every division. The analysis demonstrated that stimulated Atg $5^{-1-} \mathrm{T}$-cells did not produce daughter cells after three days and the authors concluded that Atg5 is necessary for T-cell proliferation. However, CFSE cannot differentiate between a dead or permeabilized cell and a non-proliferating cell, and over the three day period there was a moderate increase in apoptosis $[14,15]$. In this model, it appears that autophagy is required during T-cell hematopoiesis, at least in part, to prevent death of the newly differentiated cells. It would be interesting to investigate this in an inducible model, to test whether the dependence on autophagy is transient, i.e. only during early differentiation stages, or required to maintain the differentiated population.

\subsection{Adipocytes}

Evidence also supports a role for autophagy in adipocyte differentiation. Treatment of mouse embryonic fibroblasts (MEFs) with a cocktail of differentiation-inducing factors over the course of two weeks causes accumulation of lipid analogous to mature adipocytes. In the absence of Atg5 the MEFs fail to accumulate lipid droplets or display the molecular or structural phenotype of differentiated adipocytes. Furthermore, Atg $5^{-/-}$mouse pups have far fewer adipocytes [16]. Knocking down Atg7 in 3T3-L1 preadipocytes attenuated the accumulation of triglycerides and several markers of mature adipocytes as well. This was further validated in vivo in an adipose-specific Atg7 knock out mouse; the total fat in the knock out mouse weighed less than half that of the wild type mice. Interestingly, the adipose-specific Atg7-/ mouse had increased amounts of brown adipose tissue (BAT) and less white adipose tissue (WAT) relative to the wild type mouse, and both the lipid droplets and adipocytes were smaller and more densely packed than normal adipocytes $[17,18]$. The brown appearance of BAT is due to high quantities of densely packed mitochondria. BAT can uncouple the mitochondrial ATPase and continue oxidizing nutrients to generate thermal energy in place of the chemical energy provided by ATP. Zhang et al. validated these results, but went further and confirmed an increased presence of mitochondria [18]. Thus, while autophagy deficient adipocytes fail to express differentiation markers and lack lipid droplet morphology, some of the effects seen in autophagy-deficient adipocytes may result from the accumulation of mitochondria. Indeed, 
increased lipid catabolism in the form of $\beta$-oxidation occurred in the Atg $7^{--}$adipocytes [17]. These findings points to a potentially functionally significant role for mitophagy during adipocyte differentiation. WAT primarily stores fatty acids for use under starvation conditions. Perhaps mitophagy is a necessary step for preventing wasteful energy expenditure through lipid catabolism in adipocytes. However, loss of the pro-mitophagic protein Bnip3 causes hepatocytes to accumulate mitochondria like adipocytes, but at the same time actually increase anabolic processes such as fatty acid synthesis [19]. It would be interesting to identify the cause of these differences.

\subsection{Epithelial cells}

Autophagy plays a role in different aspects of epithelial differentiation. One aspect resembles that seen in erythrocytes. Keratinocyte basal cells begin differentiation by detaching from the basement membrane and moving apically toward their fate of cornification and exfoliation. In the granulation stage, keratinocytes lose their organelles and nucleus [20]. Increased proteasome activity accompanies granulation and accounts for some of the degradative action, but entire organelles may require the autophagy pathway. Granulating keratinocytes are packed with lysosome bodies, and examination of expression patterns during differentiation revealed increased expression of the pro-autophagic proteins Beclin and Sirt1 [21,22]. Whether these events are more specifically controlled by organelle-specific autophagy mechanisms as is observed in erythrocytes has yet to be determined.

A question that remains is whether there is a role for autophagy in earlier epithelial differentiation stages, such as during the first emergence of suprabasal cells in striated epithelium. In one study, 3-MA-treated keratinocytes failed to express the differentiation marker involucrin when stimulated to differentiate in low glucose and high calcium [22]. However, this study should be interpreted with caution, because the reduction in glucose used to induce differentiation is a metabolic stressor. Because the metabolic state of cells regulates rates of autophagy, the increase in autophagy seen here may be an independent coincidence caused by the metabolic stress used to induce differentiation. Additionally, the sole use of 3-MA to inhibit autophagy brings up the question of selectivity. 3-MA also inhibits class I PI3K [23], an important regulator of keratinocyte differentiation [24,25].

The most striking link between autophagy and early epithelial differentiation comes from studies in the mammary gland. Autophagy promotes the survival of differentiated breast epithelial cells in 3D models of breast acinar formation [26]. Like keratinocytes, the breast epithelial cells lose adhesion to the ECM and push away from the basement membrane as differentiation occurs. Autophagy is increased in the differentiating cells in the center of the acini. However, inhibiting autophagy with 3-MA during differentiation increases luminal cell caspase-3 cleavage and death rather than blocking differentiation per se [27]. This suggests autophagy controls the rate of lumen cell loss, but the reason for needing this intermediate step is not clear.

Another example of autophagy in epithelial differentiation is in development of the embryoid body (EB). Embryoid bodies develop spontaneously from cultured embryonic stem cells, forming a spherical body of cells that can differentiate into various cell types [28]. The inner 
cells of the EB display increased amounts of autophagic vacuoles similar to the breast epithelia 3D acini. However, unlike what happens in the breast acini, autophagy inhibition resulted in the accumulation of dead cell bodies in the EB lumen, suggesting that in this case increased autophagy is responsible for clearing out the dead cell debris accumulating at the center of the EB spheroid. In EB, cavitation occurs in response to increased hypoxia. Qi et al concluded that the hypoxic environment increased apoptosis inducing factor (AIF), which in turn increased ROS production and HIF2 $\alpha$ signaling, and subsequently up-regulated pro-autophagic Bnip3. Knocking down any of the aforementioned proteins delayed cavitation of the EB. However, unlike the 3D breast acini model, inhibiting autophagy delayed caspase cleavage [29].

The examples in breast and embryoid bodies indicate that one of the roles of autophagy is to control when and how cell death occurs during differentiation, whereas autophagy is required for organelle clearing and survival in granulocytic keratinocytes and erythrocytes [21]. Thus, depending on the situation or cell state, autophagy can play distinct roles in differentiation.

A key question that remains in many of these models is why autophagy is important during differentiation. Mitochondrial clearance is likely important for the functional aspect of erythrocytes; if erythrocytes still contained mitochondria and carried out oxidative phosphorylation they would probably deplete much of their own oxygen in circulation before reaching tissues needing oxygen. Failure to eliminate mitochondria during adipocyte differentiation results in increased catabolism and a poor ability to store fat. However, in many systems autophagy seems to allow the cells to simply survive differentiation. Perhaps the metabolic stress of differentiating takes a toll on the integrity of the mitochondria and other intracellular components and autophagy increases just to keep up with the increased demand for maintenance. On the other hand, autophagy may have specific tumor suppressive effects during differentiation. Loss or reduction in autophagy appears to be required for tumor initiation and it is well established that tumorigenesis is linked with aberrant differentiation. One possibility is that autophagy actually promotes terminal differentiation, creating an antagonist to tumorigenesis. The sacrifice the tumor cells make is to create a greater dependence on antiapoptotic survival pathways to compensate for the lack of the survival benefit of autophagy. However, as cancer progresses, some aspects of the autophagy pathway re-emerge as a mechanism to escape death under stress, but this must be balanced by preventing differentiation and reduced dependence on mitochondria for energy.

\section{Adhesion and regulation of autophagy}

In the case of multilayered epithelia, such as skin, bladder, prostate, and breast, differentiation occurs as the cells detach from the basement membrane and ascend apically. Such major changes require extensive reprogramming of signaling networks and gene expression, and cells must eliminate or inhibit the former cellular programming machinery. Failure to efficiently modify the programming can cause cellular stress and/or oncogenesis. Perhaps an immediate increase in autophagy aids in temporal separation of the undifferentiated and differentiated cell signaling and programming pathways. Coincident with this process is the 
need to maintain cell survival. This brings to question what signaling molecules in differentiation regulate autophagy.

\subsection{Integrins and detachment-induced autophagy}

Integrins, a family of heterodimeric transmembrane proteins, mediate cell adhesion to the extracellular matrix (ECM). Integrins regulate a variety of functions at the cellular and molecular level [30]. In stratified epithelial cell differentiation the integrins holding basal cells to the basement membrane become internalized and eliminated as the cell stratifies. Integrins form focal points with complexes of signaling molecules to maintain the connection between the matrix and the contracting cytoskeleton [31]. Cell survival is a key function of integrins. Loss of cell adhesion due to disengagement of integrins from their ECM ligand simultaneously activates extrinsic and intrinsic apoptotic cell death termed anoikis [32]. This signaling pathway prevents exfoliated or damaged cells from surviving detachment and adhering at an improper location.

Detachment and anoikis also aids in lumen clearing in secretory epithelium. As epithelial cells become contact inhibited, they force some cells off the basement membrane [33]. In undifferentiated epithelium this loss of adhesion would normally trigger anoikis and apoptosis. However, in differentiating epithelium, autophagy is triggered which delays the rapid onset of anoikis. This was demonstrated in both 3D acini and in suspended cells. Autophagy was induced in a subpopulation of human mammary epithelial MCF10A and canine kidney epithelial cells when they were placed in suspension, which allowed them to survive anoikis. Inhibiting autophagy by knocking down the autophagy proteins ATG5, ATG6, and ATG7 in suspended MCF-10A cells increased cleaved caspase-3 positive cells and decreased replating efficiency [27]. Further studies demonstrated that the decision to undergo autophagy, as opposed to anoikis, is controlled in part by up-regulation PERK to suppress ROS, through activation of the ER stress pathway, and resulting in increased expression of ATG6 and ATG8 [34]. When squamous cell carcinomas (SCCs) were isolated from FAK-/- mice (which presumably have altered integrin-based adhesion), or when SCCs isolated from wild type mice were placed in suspension, the rate of autophagy was significantly increased. This resulted in the targeting of Src to autophagosomes through c-Cbl in an E3-ligase-independent mechanism [35]. The major conclusion drawn from these studies is that loss of matrix adhesion induces autophagy.

\subsection{Attachment-induced autophagy}

This latter conclusion is partially contradicted by several studies demonstrating that adhesion to matrix is required to promote autophagy. In a study on human primary basal prostate epithelial cells (PrEC) researchers found that blocking integrin interactions with the ECM inhibited autophagy induced by starvation. In culture, PrECs secrete and adhere to a laminin 5, via integrins $\alpha 3 \beta 1$ and $\alpha 6 \beta 4$. These integrins were necessary for maintaining cell survival through Src and ligand-independent EGFR activation. Blocking integrin $\alpha 3, \alpha 6$, or $\beta 4$ with antibodies inhibited both survival and autophagy in these cells. Particularly interesting is that inhibiting autophagy with 3-MA or blocking integrin $\alpha 3$ antibodies induced caspase cleavage, 
but inhibiting EGFR alone did not. Since a class I-specific inhibitor could not induce apoptosis in PrECs, it is unlikely that off target inhibition of class I PI3K by 3-MA resulted in caspase cleavage. In addition, cell adhesion or inhibition of EGFR had no effect on Akt phosphorylation, which suggests that integrin-mediated maintenance of autophagy does not occur through the PI3K/Akt pathway in PrECs [36]. This study demonstrated that integrin adhesion mediates autophagy. While the exact downstream signaling pathway from integrins to autophagy remains unclear, survival was dependent on Erk signaling which is known to promote autophagy. A subsequent study demonstrated that in prostate cancer cells the androgen receptor promoted survival via integrin $\alpha 6 \beta 1$ activation of IKK/NF- $\kappa \mathrm{B}$ signaling, independent of the PI3K/Akt pathway [37]. A similar dependence on the IKK/NF- $\kappa B$ pathway for survival is mediated by integrin $\alpha 6 \beta 4$ in polarized cells of 3D breast acinar structures and over activation of this pathway is sufficient to overcome suspension-induced anoikis and apoptosis [38-40]. Given that activation of the IKK/NF- $\kappa B$ pathway can induce autophagy [41], it is reasonable to suspect that integrins may regulate autophagy in part through the IKK/NF- $\mathrm{B}$ pathway (Figure 1A). Further insight into how integrins regulate NF- $\kappa \mathrm{B}$ and autophagy is needed.

Stimulation of smooth muscle cells with the integrin ligand osteopontin, stimulated autophagy related-genes, autophagosome formation, and ultimately cell death [42]. This effect was mediated by p38-MAPK through integrin and CD44 (Figure 1C). In a Drosophila genetic screen, paxillin, a downstream target of integrins, was found to associate with Atg1 and to be required for starvation-induced autophagy in MEFs [43]. However, in the fly, the ability of paxillin to facilitate autophagy was not linked with integrins. Whether this is true in the mammalian model has not been determined. Thus, several studies implicate integrin-based adhesion in both suppressing as well as stimulating autophagy. Since all these studies were conducted in different model systems and under different culture conditions, it is difficult to draw a definitive conclusion. Therefore, when studying different biological events it is necessary to consider several possible mechanisms and signaling pathways that could influence autophagy.

Some consideration should be given to a potential alternative mechanism to explain detachment-induced autophagy. Although it was quite thoroughly demonstrated that the cells increased the rate of autophagy after losing ECM contact, in both the EB and acini models the cells are undergoing a differentiation program and remaining in contact with neighboring cells. In the MCF-10A breast model where cells were put into suspension and $25 \%$ survived anoikis, these cells existed in large aggregates [27]. In light of the fact that increased cell density also increases autophagy in vitro, one could hypothesize that the proteins mediating cell-cell contact may be signaling to increase autophagy in the detached cells [23].

\subsection{Cell-cell adhesion-induced autophagy}

A relatively new process called entosis, whereby cell-cell adhesion induces the entry of one cell into a neighboring cell, was found to trigger an autophagic response [44] by mechanisms that resemble pathogen engulfment by immune cells. The ability of cells to entose depends on adherens junctions in the absence of integrin adhesion [45]. The major protein that mediates 
A

\section{B}
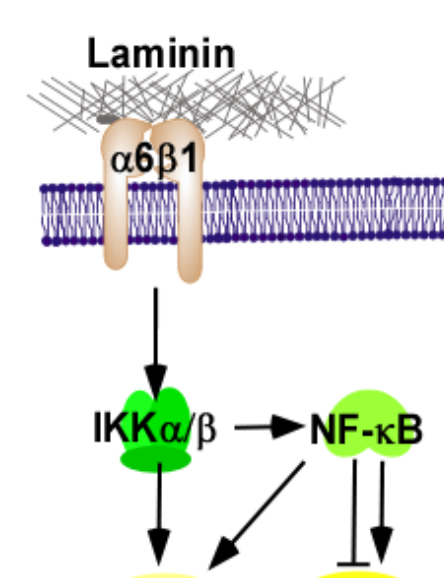

\section{E-Cadherin} Beclin1
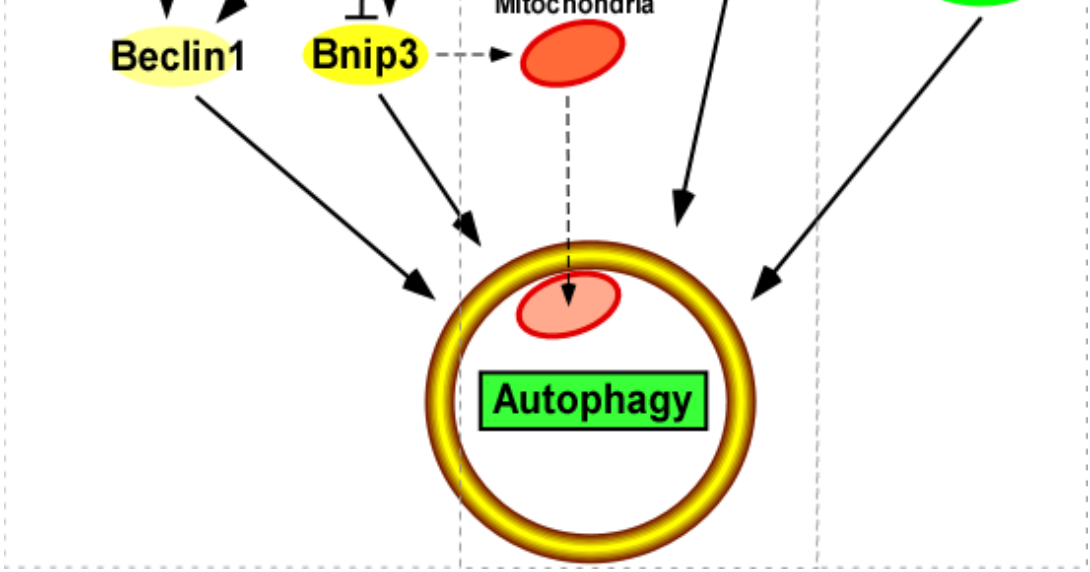

Mitochondria

Figure 1. Cell Adhesion Pathways that Promote Autophagy. (A) Integrin a6 $\beta 1$ activates NF-KB through IKK signaling. Together, IKK and NF-KB can up-regulate and activate Beclin, and NF-KB in turn regulates pro-mitophagic Bnip3 expression. (B) AMPK localizes with LKB1 at adherens junctions where LKB1 activates AMPK signaling, which can activate autophagy through Ulk1. (C) Osteopontin activates p38 MAPK-induced autophagy through CD44 and av $\beta 3$.

epithelial cell-cell contact through adherens junctions is E-cadherin. This vital protein holds the epithelium together and promotes the survival of cells [46-48]. Adherens junctions mediate the connection between cells by essentially bridging the actin cytoskeleton of two different cells. A plethora of signaling events are controlled by E-cadherin. In the case of autophagy, one point of interest is regulation of AMPK.

In response to low ATP levels, AMPK directly phosphorylates and activates Ulk1 in parallel to deactivating the autophagy suppressor mTOR [49]. Membrane bound E-cadherin localizes with the AMPK activator LKB1 (Figure 1B), and by doing so increases AMPK activation by bringing it into close proximity with LKB1 [50]. In one study AMPK was required for the drug 
Atorva to induce an endoplasmic reticulum stress response, autophagy, and phosphorylation of eIF2 $\alpha$ [51]. Interestingly, in the MCF-10A detachment-induced autophagy model, suspended cells had increased levels of activated PERK, a well-documented cytoprotective ER stressresponse protein that induces autophagy, and increased phosphorylation of the downstream PERK target eIF2 $\alpha$ in conjunction with increased autophagic flux [34]. Considering the overlapping pathways and the correlation of detachment-induced autophagy with cell-cell contact this could be a promising area to investigate.

\subsection{Genetic evidence for integrin adhesion and autophagy}

Deficiency of the laminin $\alpha 2$ chain (LAMA2) in laminin 2 causes a form of congenital muscular dystrophy known as MDC1A. MDC1A patients present with severe muscle weakness, peripheral neuropathy, and joint contractures [52]. The LAMA2-deficient mouse model $\left(\mathrm{dy}^{3 \mathrm{~K}} /\right.$ $\mathrm{dy}^{3 \mathrm{~K}}$ ) develops muscle weakness two weeks after birth and dies by 5 weeks with severe muscular dystrophy [53,54]. The $\mathrm{dy}^{3 \mathrm{~K}} / \mathrm{dy}^{3 \mathrm{~K}}$ mice feature increases in myocyte apoptosis, degeneration/regeneration cycles, variable fiber sizes, and connective tissue hyperproliferation. $\mathrm{dy}^{3 \mathrm{~K}} / \mathrm{dy}^{3 \mathrm{~K}}$ mice exhibit significantly increased expression of the autophagy related genes Bnip3, Bnip31, p62, LC3B, GABARAP1, Vps34, Atg4b, Cathepsin L, Lamp2a, and Beclin. Administration of 3-MA to the $\mathrm{dy}^{3 \mathrm{~K}} / \mathrm{dy}^{3 \mathrm{~K}}$ mice increased lifespans, increased average muscle fiber diameter, decreased the presence of caspase- 3 in myocytes, and partially restored muscular morphology. In addition, immunofluorescent staining of muscle biopsies from MDC1A patients showed an increased amount of LC3B in the myocytes. Thus, patients with MDC1A may essentially suffer from excessive muscular autophagy due to a lack of interaction with the matrix protein laminin 2 (Figure 2A). This example supports a role for matrix detachment in inducing autophagy.

On the other hand, one of the LAMA2 receptors, dystroglycan which binds laminin extracellularly and dystrophin intracellularly, may promote adhesion-dependent autophagy. One function of the dystroglycan/dystrophin complex is to bind F-actin to anchor the cytoskeleton in place. Like the $\mathrm{dy}^{3 \mathrm{~K}} / \mathrm{dy}^{3 \mathrm{~K}}$ mice, the dystrophin mutant $(\mathrm{mdx})$ mice display a muscular dystrophy phenotype. However, in this case, the $\mathrm{mdx}$ mice accumulate damaged mitochondria, and inducing autophagy through AMPK activation in these mice ameliorates the disease phenotype, indicating there is a defect in muscle autophagy in mdx mice. This further suggests that adhesion to laminin through the dystroglycan/dystrophin connection is required to maintain autophagy in myocytes [55,56]. It is worth noting that mutations in integrin $\alpha 7$ are associated with muscular dystrophy as well. The integrin dimer $\alpha 7 \beta 1$ is another receptor that binds laminin- $\alpha 2$ and resides on myocytes [57]. However, whether $\alpha 7 \beta 1$ interactions affect autophagy in these diseases remains unknown. Further evidence supporting the concept that cell adhesion mediates autophagy comes from the study of Ulrich's Congenital Muscular Dystrophy (UCMD) and Bethlem Myopathy. Both diseases involve chronic weakening and degradation of skeletal muscle due to the spontaneous death of myocytes, which ultimately leads to death. Genetic studies discerned that mutations in the gene coding for collagen VI correlate with the condition [58]. Furthermore, genetically modified mice lacking the collagen VI gene (Col6-/-) develop myopathies similar to UCMD and Bethlem Myopathy. The Col6-- 
A

Laminin $\alpha 2$

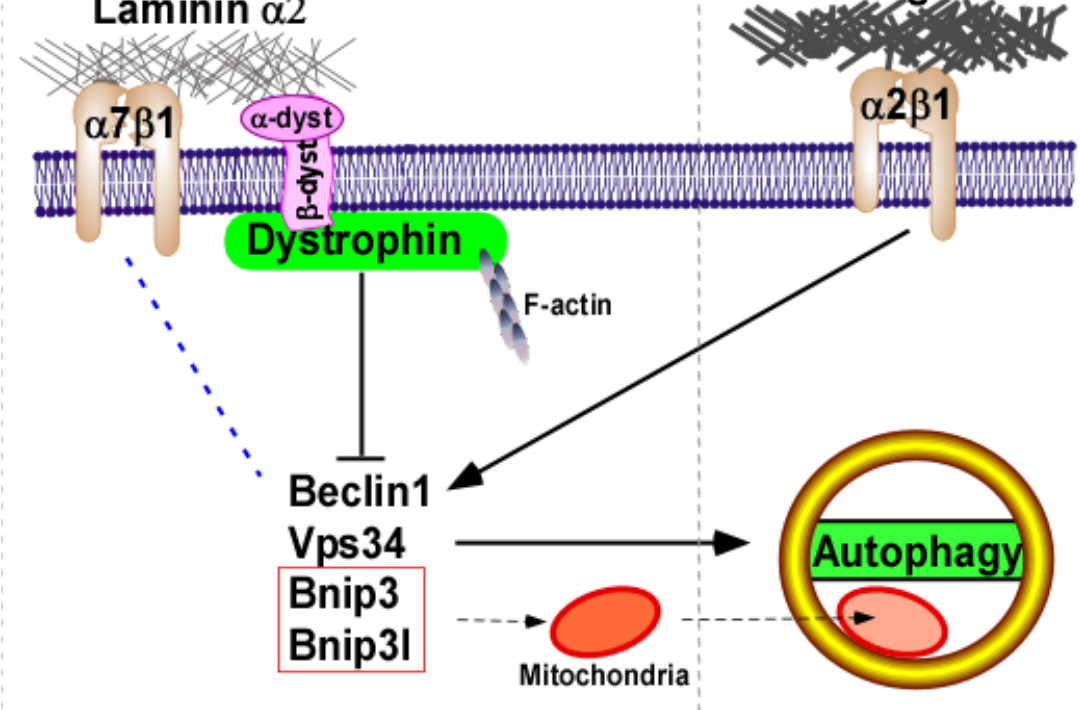

Figure 2. Matrix Adhesion Pathways and Autophagy in Muscle Cells. (A) Laminin a2 is bound by the $a-$ and $\beta$-dystroglycan complex, which in turn binds dystrophin, a protein that anchors the complex onto F-actin. Loss of Laminin a2 caused increase expression of Beclin, Bnip3, Bnip3I, Vps34, and several other autophagy related proteins. Both Bnip3 and Bnip3I can induce mitophagy as well. Integrin a7 $\beta 1$ binds laminin a 2 as well, but a connection with autophagy has not been established. (B) Loss of Collagen VI increased pro autophagic and pro mitophagic genes and caused decreased autophagy along with decreased expression of Beclin and Bnip3. The collagen receptor integrin a $2 \beta 1$ may be responsible for this.

mice have dysmorphic and dysfunctional myofibers characterized by increased apoptosis and reduced muscle strength compared to wild type mice [59]. Electron microscopy of Col6-1myocytes revealed damaged and misshapen mitochondria and biochemical assays demonstrated that the mitochondria are dysfunctional. Inhibiting mitochondrial permeablization with Cyclosporine $\mathrm{A}$ in $\mathrm{Col}^{-/}$mice and in patients with homologous myopathies ameliorated the diseases effects [60], and genetic ablation of the cyclosporine A target, Cyclophilin D, also mitigated the symptoms in $\mathrm{Col}^{-/}$mice [61]

Later research found that Col6-- mice muscle tissue expressed lower levels of the pro-autophagic proteins Beclin and Bnip3 and displayed a significant decrease in autophagy (Figure 2B). Forced expression of Beclin and Bnip3 partially reversed the disease phenotype in $\mathrm{Col}^{-/}$myocytes, and inducing autophagy by fasting the Col6- mice reestablished autophagy and decreased apoptosis in the myocytes. Furthermore, fasting or treating the $\mathrm{Col}^{-/}$mice with Rapamycin, to block the autophagy inhibitor mTor, decreased the presence of dysfunctional mitochondria [62]. These latter studies on Col6-related myopathies indicate that myocyte cell adhesion to collagen maintains autophagic flux and cell survival, while unregulated detachment inhibits autophagy. 


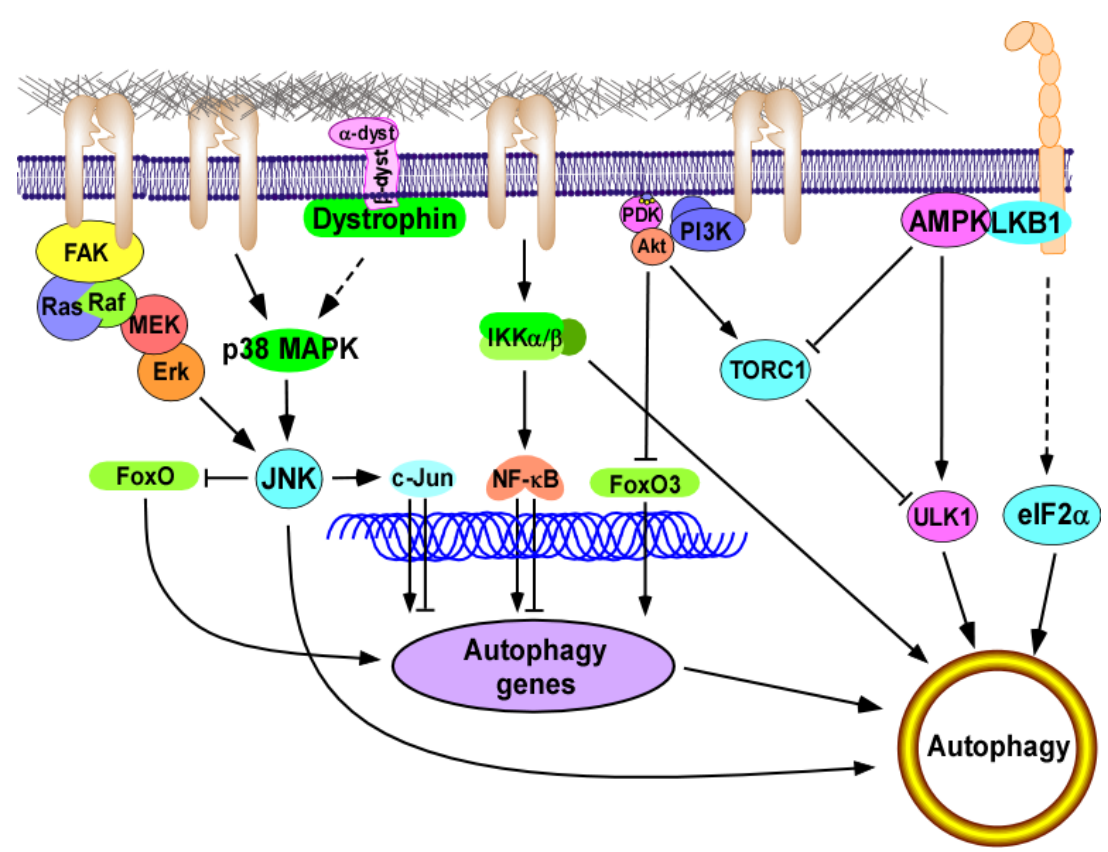

Figure 3. Mechanisms for Cell Adhesion-Induced Autophagy. The intracellular domain of integrins binds to FAK, which activates the Ras/Raf/MEK/Erk signaling cascade. Integrins and dystrophin both can activate p38 MAPK. Both Erk and p38 can phosphorylate JNK and induce autophagy. JNK in turn can either inhibit autophagy through suppression of FoxO or promote autophagy through activation of c-Jun, which can positively and negatively regulate autophagy. Integrins can also activate the IKKa/ $\beta-N F-K B$ pathway. IKKa/ $\beta$ induces autophagy both dependently and independently of NF-KB; however, NF-кB can also inhibit autophagy in some cases. Integrins also activate the PI3K/Akt/TORC1, which inhibit autophagy by phosphorylating and inhibiting FoxO3 and UIK1. LKB1 binds to E-cadherin and activates AMPK. AMPK both activates Ulk1 and inhibits TORC1, causing an increase in autophagy. Some evidence indicates that cell-cell adhesion may also activate the autophagy-inducer elF2a.

\subsection{Cell adhesion signaling pathways that regulate autophagy}

Adhesion proteins interact with a host of proteins and activate several well characterized pathways that affect autophagy. Recall that the dystrophin glycoprotein complex promotes adhesion dependent signaling in myocytes, and the importance of this was highlighted in the $\mathrm{Mdx}$ and $\mathrm{dy}^{3 \mathrm{~K}} / \mathrm{dy}^{3 \mathrm{~K}}$ mice (Figure $2 \mathrm{~A}$ ). When the dystrophin glycoprotein complex is bound to laminin, an intracellular component of the complex called syntrophin binds and activates the Rac1 signaling complex, leading to activation of JNK, a protein that can both positively and negatively regulate autophagy [63] (Figure 3). JNK can induce autophagy by phosphorylating Bcl-2 and thus preventing Bcl-2 from inhibiting Beclin [64]. JNK also regulates several transcription factors that in turn mediate expression or repression of autophagy related genes. For example, genetic ablation of all three JNK isoforms results in constitutive activation of the transcription factor FoxO, causing an increase in autophagy and the effector proteins Bnip3 and Beclin. JNK can also activate the transcription factor c-Jun, a protein which when overex- 
pressed can inhibit autophagy under starvation conditions [65]. On the other hand, another study showed that JNK was required for ceramide induced autophagy and expression of Beclin [66]. Furthermore, Jnk1 can phosphorylate Bcl-2 to release Beclin and promote autophagy [67]. Integrins may also regulate autophagy via 338 MAPK. Osteopontin binds integrins and CD44 and induces autophagy through activation of p38 MAPK [42]. Although some evidence indicates that p38 inhibits autophagy, p38 can activate JNK [68], which could provide a potential mechanism for both positive and negative regulation of autophagy by integrins. Finally, integrins stimulate Erk1/2 signaling as well, which activates autophagy through numerous pathways including activation of JNK [68].

Signaling through IKK $\alpha / \beta-\mathrm{NF}-\kappa \mathrm{B}$ is another node for promoting autophagy (Figure 3). IKK $\alpha /$ $\beta$ regulates the expression of autophagy-related genes by activating NF- $\kappa B$, which in turn has been shown to both positively and negatively regulate autophagy $[41,69,70]$. IKK $\alpha / \beta$ can also activate autophagy independently of NF- $\mathrm{kB}$ [70]. Cells expressing constitutively active subunits of the IKK complex had increased autophagy and increased levels of phosphorylated active AMPK and JNK [71]. Considering that blocking integrin adhesion in PrECs subsequently attenuated autophagy [36], and that integrin mediated adhesion to laminin increased IKK $\alpha$ / $\beta$ - NF- $\kappa B$ signaling enough to significantly increase survival in a prostate cancer line [46], signaling through IKK $\alpha / \beta$ - NF- $\kappa B$ may be a mechanism by which integrin-mediated-celladhesion promotes autophagy. It would be interesting to determine whether this mechanism occurs and whether it is dependent or independent of NF- $\kappa \mathrm{B}$.

PI3K/Akt signaling potently suppresses autophagy [72,73] (Figure 3). Akt activates the TORC1 complex, which in turn phosphorylates and inactivates the autophagy-initiating kinase Ulk1 [73]. Akt itself phosphorylates and inactivates the FoxO3a transcription factor. This prevents FoxO3a from inducing the expression of numerous autophagy- and Mitophagy-related genes [74]. Integrins can activate PI3K/Akt signaling [30], making it possible that integrins could suppress autophagy through this pathway as well. Perhaps this explains the cases in which detachment from the basement membrane actually promoted autophagy. However, it was shown that detachment of breast epithelial cells both increased autophagy and induced phosphorylation of eIF2 $\alpha$, a target of the ER kinase PERK and a protein capable of inducing autophagy [34].

AMPK activates autophagy by inhibiting TORC1 and activating Ulk1 via direct phosphorylation [49]. LKB1 localizes with engaged E-cadherin and activates AMPK signaling when ATP levels are low (Figure 3), providing a potential mechanism for cell-cell-interaction-mediated autophagy that might be relevant in detachment induced autophagy mechanisms in which cell-cell contact is maintained [50].

\section{Conclusion}

In many of the studies discussed above and in other diseases and models the effects of autophagy are very mitochondria-centric. For example, the $\mathrm{Col}^{-/}$mice phenotype was rescued by inhibiting mitochondrial depolarization, re-expressing Beclin or the pro- 
mitophagic protein Bnip3, or by starvation-induced autophagy [61,62]. Although not discussed in this chapter, it is interesting to note that Parkinson's disease is associated with mutations in several genes that target damaged mitochondria for mitophagy, and increasing evidence implicates mitophagy as having a role in retarding aging in general $[75,76]$. Considering that mitochondria can induce apoptosis and regulate the levels of ROS, one could imagine mitophagy as being a mechanism of survival during detachment or a method of maintaining cell integrity during adhesion.

Cell interaction with ECM and other cells of the same organism is imperative for - and essentially defines - multicellular organisms. Aside from being the scaffolding that organizes cells into functional macrostructures, the ECM extensively regulates cellular behavior [30]. The importance of these interactions is well demonstrated in the mounting evidence indicating that varied interactions with the ECM can actually differentially dictate the fate of stem cell differentiation [77]. Understanding the effects of the ECM on cell biology not only increases the understanding of normal organismal development and biology, but also diseases such as cancer or the various forms of muscular dystrophy $[78,79]$. With the current rise in understanding of autophagy in diseases and development, understanding how extracellular interactions and differentiation affect autophagy is an important avenue to explore in the future.

\section{Nomenclature}

3-MA - 3-methyladenine

AIF - apoptosis inducing factor

BAT - brown adipose tissue

Col6 - collagen VI gene

CFSE - 5,6-carboxyfluorescein diacetate succinimidyl ester

EB - embryoid body

ECM - extracellular matrix

LAMA2 - laminin $\alpha 2$ chain

Mdx - dystrophin gene

MEF - mouse embryo fibroblast

PrEC - prostate epithelial cell

SSC- squamous cell carcinomas

UCMD - Ulrich's Congenital Muscular Dystrophy

WAT- white adipose tissues 


\section{Acknowledgements}

We wish to thank the Van Andel Research Institute (EAN, CKM) and the Van Andel Institute Graduate School (EAN) for their generous support. This work was also supported in part by an NIH/NCI grant CA154835 (CKM).

\section{Author details}

Eric A. Nollet ${ }^{1,2}$ and Cindy K. Miranti ${ }^{{ }^{*}}$

*Address all correspondence to: cindy.miranti@vai.org

1 Laboratory of Integrin Signaling and Tumorigensis, Van Andel Research Institute, Grand Rapids, Michigan, USA

2 Van Andel Institute Graduate School, Grand Rapids, Michigan, USA

\section{References}

[1] Gronowicz, G, Swift, H, \& Steck, T. L. Maturation of the reticulocyte in vitro. J Cell Sci. (1984). http://www.ncbi.nlm.nih.gov/pubmed/6097593, 71, 177-97.

[2] Koury, M. J, Koury, S. T, Kopsombut, P, \& Bondurant, M. C. In vitro maturation of nascent reticulocytes to erythrocytes. Blood. (2005). http://www.ncbi.nlm.nih.gov/ pubmed/15528310, 105(5), 2168-74.

[3] Kundu, M, Lindsten, T, Yang, C. Y, Wu, J, Zhao, F, Zhang, J, et al. Ulk1 plays a critical role in the autophagic clearance of mitochondria and ribosomes during reticulocyte maturation. Blood. (2008). http://www.ncbi.nlm.nih.gov/pubmed/18539900, 112(4), 1493-502.

[4] Schweers, R. L, Zhang, J, Randall, M. S, Loyd, M. R, Li, W, Dorsey, F. C, et al. NIX is required for programmed mitochondrial clearance during reticulocyte maturation. Proc Natl Acad Sci U S A. (2007). http://www.ncbi.nlm.nih.gov/pubmed/18048346, 104(49), 19500-5.

[5] Ney, P. A. Normal and disordered reticulocyte maturation. Curr Opin Hematol. (2011). http://www.ncbi.nlm.nih.gov/pubmed/21423015, 18(3), 152-7.

[6] Zhang, J, Loyd, M. R, Randall, M. S, Waddell, M. B, Kriwacki, R. W, \& Ney, P. A. A short linear motif in BNIP3L (NIX) mediates mitochondrial clearance in reticulocytes. Autophagy. (2012). http://www.ncbi.nlm.nih.gov/pubmed/22906961, 8(9), 1325-32. 
[7] Zhang, J, Randall, M. S, Loyd, M. R, Dorsey, F. C, Kundu, M, Cleveland, J. L, et al. Mitochondrial clearance is regulated by Atg7-dependent and-independent mechanisms during reticulocyte maturation. Blood. (2009). http://www.ncbi.nlm.nih.gov/ pubmed/19417210, 114(1), 157-64.

[8] Aerbajinai, W, Giattina, M, Lee, Y. T, Raffeld, M, \& Miller, J. L. The proapoptotic factor Nix is coexpressed with Bcl-xL during terminal erythroid differentiation. Blood. (2003). http://www.ncbi.nlm.nih.gov/pubmed/12663450, 102(2), 712-7.

[9] Hanna, R. A, Quinsay, M. N, Orogo, A. M, Giang, K, Rikka, S, \& Gustafsson, A. B. Microtubule-associated protein 1 light chain 3 (LC3) interacts with Bnip3 protein to selectively remove endoplasmic reticulum and mitochondria via autophagy. J Biol Chem. (2012). http://www.ncbi.nlm.nih.gov/pubmed/22505714, 287(23), 19094-104.

[10] Cebollero, E, Reggiori, F, \& Kraft, C. Reticulophagy and ribophagy: regulated degradation of protein production factories. Int J Cell Biol. (2012). http:// www.ncbi.nlm.nih.gov/pubmed/22481944

[11] Mortensen, M, Ferguson, D. J, Edelmann, M, Kessler, B, Morten, K. J, Komatsu, M, et al. Loss of autophagy in erythroid cells leads to defective removal of mitochondria and severe anemia in vivo. Proc Natl Acad Sci U S A. (2010). http:// www.ncbi.nlm.nih.gov/pubmed/20080761, 107(2), 832-7.

[12] Pua, H. H, Guo, J, Komatsu, M, \& He, Y. W. Autophagy is essential for mitochondrial clearance in mature T lymphocytes. J Immunol. (2009). http://www.ncbi.nlm.nih.gov/ pubmed/19299702, 182(7), 4046-55.

[13] Stephenson, L. M, Miller, B. C, Ng, A, Eisenberg, J, Zhao, Z, Cadwell, K, et al. Identification of Atg5-dependent transcriptional changes and increases in mitochondrial mass in Atg5-deficient $\mathrm{T}$ lymphocytes. Autophagy. (2009). http:// www.ncbi.nlm.nih.gov/pubmed/19276668, 5(5), 625-35.

[14] Dumitriu, I. E, Mohr, W, Kolowos, W, Kern, P, Kalden, J. R, \& Herrmann, M. 5,6-carboxyfluorescein diacetate succinimidyl ester-labeled apoptotic and necrotic as well as detergent-treated cells can be traced in composite cell samples. Anal Biochem. (2001). http://www.ncbi.nlm.nih.gov/pubmed/11730350, 299(2), 247-52.

[15] Pua, H. H, Dzhagalov, I, Chuck, M, Mizushima, N, \& He, Y. W. A critical role for the autophagy gene Atg5 in T cell survival and proliferation. The Journal of experimental medicine. (2007). http://www.ncbi.nlm.nih.gov/pubmed/17190837, 204(1), 25-31.

[16] Baerga, R, Zhang, Y, Chen, P. H, Goldman, S, \& Jin, S. Targeted deletion of autophagy-related 5 (atg5) impairs adipogenesis in a cellular model and in mice. Autophagy. (2009). http://www.ncbi.nlm.nih.gov/pubmed/19844159, 5(8), 1118-30.

[17] Singh, R, Xiang, Y, Wang, Y, Baikati, K, Cuervo, A. M, Luu, Y. K, et al. Autophagy regulates adipose mass and differentiation in mice. The Journal of clinical investigation. (2009). http://www.ncbi.nlm.nih.gov/pubmed/19855132, 119(11), 3329-39. 
[18] Zhang, Y, Goldman, S, Baerga, R, Zhao, Y, Komatsu, M, \& Jin, S. Adipose-specific deletion of autophagy-related gene 7 (atg7) in mice reveals a role in adipogenesis. Proc Natl Acad Sci U S A. (2009). http://www.ncbi.nlm.nih.gov/pubmed/19910529, 106(47), 19860-5.

[19] Glick, D, Zhang, W, Beaton, M, Marsboom, G, Gruber, M, Simon, M. C, et al. BNip3 regulates mitochondrial function and lipid metabolism in the liver. Mol Cell Biol. (2012). http://www.ncbi.nlm.nih.gov/pubmed/22547685, 32(13), 2570-84.

[20] Lippens, S, Denecker, G, Ovaere, P, Vandenabeele, P, \& Declercq, W. Death penalty for keratinocytes: apoptosis versus cornification. Cell Death Differ. (2005). Suppl http://www.ncbi.nlm.nih.gov/pubmed/16247497, 2, 1497-508.

[21] Gosselin, K, Deruy, E, Martien, S, Vercamer, C, Bouali, F, Dujardin, T, et al. Senescent keratinocytes die by autophagic programmed cell death. Am J Pathol. (2009). http:// www.ncbi.nlm.nih.gov/pubmed/19147823, 174(2), 423-35.

[22] Aymard, E, Barruche, V, Naves, T, Bordes, S, Closs, B, Verdier, M, et al. Autophagy in human keratinocytes: an early step of the differentiation? Exp Dermatol. (2011). http://www.ncbi.nlm.nih.gov/pubmed/21166723, 20(3), 263-8.

[23] Klionsky, D. J, Abdalla, F. C, Abeliovich, H, Abraham, R. T, Acevedo-arozena, A, Adeli, K, et al. Guidelines for the use and interpretation of assays for monitoring autophagy. Autophagy. (2012). http://www.ncbi.nlm.nih.gov/pubmed/22966490, 8(4), 445-544.

[24] Xie, Z, \& Bikle, D. D. The recruitment of phosphatidylinositol 3-kinase to the E-cadherin-catenin complex at the plasma membrane is required for calcium-induced phospholipase C-gamma1 activation and human keratinocyte differentiation. J Biol Chem. (2007). http://www.ncbi.nlm.nih.gov/pubmed/17242406, 282(12), 8695-703.

[25] Calautti, E, Li, J, Saoncella, S, Brissette, J. L, \& Goetinck, P. F. Phosphoinositide 3-kinase signaling to Akt promotes keratinocyte differentiation versus death. J Biol Chem. (2005). http://www.ncbi.nlm.nih.gov/pubmed/16036919, 280(38), 32856-65.

[26] Kenny, P. A, Lee, G. Y, Myers, C. A, Neve, R. M, Semeiks, J. R, Spellman, P. T, et al. The morphologies of breast cancer cell lines in three-dimensional assays correlate with their profiles of gene expression. Molecular oncology. (2007). http:// www.ncbi.nlm.nih.gov/pubmed/18516279, 1(1), 84-96.

[27] Fung, C, Lock, R, Gao, S, Salas, E, \& Debnath, J. Induction of autophagy during extracellular matrix detachment promotes cell survival. Mol Biol Cell. (2008). http:// www.ncbi.nlm.nih.gov/pubmed/18094039, 19(3), 797-806.

[28] Desbaillets, I, Ziegler, U, Groscurth, P, \& Gassmann, M. Embryoid bodies: An in vitro model of mouse embryogenesis. Exp Physiol. (2000). http:// www.ncbi.nlm.nih.gov/pubmed/11187960, 85(6), 645-51. 
[29] Qi, Y, Tian, X, Liu, J, Han, Y, Graham, A. M, Simon, M. C, et al. Bnip3 and AIF cooperate to induce apoptosis and cavitation during epithelial morphogenesis. J Cell Biol. (2012). http://www.ncbi.nlm.nih.gov/pubmed/22753893, 198(1), 103-14.

[30] Miranti, C. K, \& Brugge, J. S. Sensing the environment: a historical perspective on integrin signal transduction. Nature cell biology. (2002). Ehttp:// www.ncbi.nlm.nih.gov/pubmed/11944041, 83-90.

[31] Sieg, D. J, Hauck, C. R, \& Schlaepfer, D. D. Required role of focal adhesion kinase (FAK) for integrin-stimulated cell migration. J Cell Sci. (1999). Pt 16):2677-91. http:// www.ncbi.nlm.nih.gov/pubmed/10413676

[32] Frisch, S. M, \& Francis, H. Disruption of epithelial cell-matrix interactions induces apoptosis. J Cell Biol. (1994). http://www.ncbi.nlm.nih.gov/pubmed/8106557, 124(4), 619-26.

[33] Mizushima, N, \& Levine, B. Autophagy in mammalian development and differentiation. Nature cell biology. (2010). http://www.ncbi.nlm.nih.gov/pubmed/20811354, 12(9), 823-30.

[34] Avivar-valderas, A, Salas, E, Bobrovnikova-marjon, E, Diehl, J. A, Nagi, C, Debnath, $\mathrm{J}$, et al. PERK integrates autophagy and oxidative stress responses to promote survival during extracellular matrix detachment. Mol Cell Biol. (2011). http:// www.ncbi.nlm.nih.gov/pubmed/21709020, 31(17), 3616-29.

[35] Sandilands, E, Serrels, B, Mcewan, D. G, Morton, J. P, Macagno, J. P, Mcleod, K, et al. Autophagic targeting of Src promotes cancer cell survival following reduced FAK signalling. Nature cell biology. [10.1038/ncb2386]. (2012). http:// www.ncbi.nlm.nih.gov/pubmed/22138575, 14(1), 51-60.

[36] Edick, M. J, Tesfay, L, Lamb, L. E, Knudsen, B. S, \& Miranti, C. K. Inhibition of integrin-mediated crosstalk with epidermal growth factor receptor/Erk or Src signaling pathways in autophagic prostate epithelial cells induces caspase-independent death. Mol Biol Cell. (2007). http://www.ncbi.nlm.nih.gov/pubmed/17475774, 18(7), 2481-90.

[37] Lamb, L. E, Zarif, J. C, \& Miranti, C. K. The androgen receptor induces integrin a6b1 to promote prostate tumor cell survival via NF-kB and Bcl-xL Independently of PI3K signaling. Cancer Res. (2011). http://www.ncbi.nlm.nih.gov/pubmed/21310825, 71(7), 2739-49.

[38] Friedland, J. C, Lakins, J. N, Kazanietz, M. G, Chernoff, J, Boettiger, D, \& Weaver, V. M. a. a6b4 integrin activates Rac-dependent kinase 1 to drive NF-kB-dependent resistance to apoptosis in 3D mammary acini. J Cell Sci. (2007). Pt 20):3700-12. http:// www.ncbi.nlm.nih.gov/pubmed/17911169, 21.

[39] Weaver, V. M, Lelievre, S, Lakins, J. N, Chrenek, M. A, Jones, J. C, Giancotti, F, et al. b4 integrin-dependent formation of polarized three-dimensional architecture confers 
resistance to apoptosis in normal and malignant mammary epithelium. Cancer Cell. (2002). http://www.ncbi.nlm.nih.gov/pubmed/12242153, 2(3), 205-16.

[40] Zahir, N, Lakins, J. N, Russell, A, Ming, W, Chatterjee, C, Rozenberg, G. I, et al. Autocrine laminin-5 ligates a6b4 integrin and activates RAC and NF-kB to mediate anchorage-independent survival of mammary tumors. J Cell Biol. (2003). http:// www.ncbi.nlm.nih.gov/pubmed/14691145, 163(6), 1397-407.

[41] Copetti, T, Bertoli, C, Dalla, E, Demarchi, F, \& Schneider, C. p. RelA modulates BECN1 transcription and autophagy. Mol Cell Biol. (2009). http:// www.ncbi.nlm.nih.gov/pubmed/19289499, 29(10), 2594-608.

[42] Zheng, Y. H, Tian, C, Meng, Y, Qin, Y. W, Du, Y. H, Du, J, et al. Osteopontin stimulates autophagy via integrin/CD44 and MAPK signaling pathways in vascular smooth muscle cells. J Cell Physiol. (2012). http://www.ncbi.nlm.nih.gov/pubmed/ $21374592,38$.

[43] Chen, G. C, Lee, J. Y, Tang, H. W, Debnath, J, Thomas, S. M, \& Settleman, J. Genetic interactions between Drosophila melanogaster Atg1 and paxillin reveal a role for paxillin in autophagosome formation. Autophagy. (2008). http:// www.ncbi.nlm.nih.gov/pubmed/17952025, 4(1), 37-45.

[44] Florey, O, Kim, S. E, Sandoval, C. P, Haynes, C. M, \& Overholtzer, M. Autophagy machinery mediates macroendocytic processing and entotic cell death by targeting single membranes. Nature cell biology. (2011). http://www.ncbi.nlm.nih.gov/ pubmed/22002674, 13(11), 1335-43.

[45] Overholtzer, M, Mailleux, A. A, Mouneimne, G, Normand, G, Schnitt, S. J, King, R. $\mathrm{W}$, et al. A nonapoptotic cell death process, entosis, that occurs by cell-in-cell invasion. Cell. (2007). http://www.ncbi.nlm.nih.gov/pubmed/18045538, 131(5), 966-79.

[46] Lamb, L. E, \& Knudsen, B. S. Miranti CK. E-cadherin-mediated survival of androgenreceptor-expressing secretory prostate epithelial cells derived from a stratified in vitro differentiation model. J Cell Sci. (2010). Pt 2):266-76. http:// www.ncbi.nlm.nih.gov/pubmed/20048343

[47] Boussadia, O, Kutsch, S, Hierholzer, A, \& Delmas, V. Kemler R. E-cadherin is a survival factor for the lactating mouse mammary gland. Mech Dev. (2002). http:// www.ncbi.nlm.nih.gov/pubmed/12049767

[48] Li, L, Bennett, S. A, \& Wang, L. Role of E-cadherin and other cell adhesion molecules in survival and differentiation of human pluripotent stem cells. Cell Adh Migr. (2012). http://www.ncbi.nlm.nih.gov/pubmed/22647941, 6(1), 59-70.

[49] Kim, J, Kundu, M, Viollet, B, \& Guan, K. L. AMPK and mTOR regulate autophagy through direct phosphorylation of Ulk1. Nature cell biology. (2011). http:// www.ncbi.nlm.nih.gov/pubmed/21258367, 13(2), 132-41. 
[50] Sebbagh, M, Santoni, M. J, Hall, B, Borg, J. P, \& Schwartz, M. A. Regulation of LKB1/ STRAD localization and function by E-cadherin. Current biology : CB. (2009). http:// www.ncbi.nlm.nih.gov/pubmed/19110428, 19(1), 37-42.

[51] Yang, P. M, Liu, Y. L, Lin, Y. C, Shun, C. T, Wu, M. S, \& Chen, C. C. Inhibition of autophagy enhances anticancer effects of atorvastatin in digestive malignancies. Cancer Res. (2010). http://www.ncbi.nlm.nih.gov/pubmed/20876807, 70(19), 7699-709.

[52] Allamand, V, \& Guicheney, P. Merosin-deficient congenital muscular dystrophy, autosomal recessive (MDC1A, MIM\#156225, LAMA2 gene coding for alpha2 chain of laminin). Eur J Hum Genet. (2002). http://www.ncbi.nlm.nih.gov/pubmed/11938437, 10(2), 91-4.

[53] Miyagoe, Y, Hanaoka, K, Nonaka, I, Hayasaka, M, Nabeshima, Y, Arahata, K, et al. Laminin a2 chain-null mutant mice by targeted disruption of the Lama2 gene: a new model of merosin (laminin 2)-deficient congenital muscular dystrophy. FEBS Lett. (1997). http://www.ncbi.nlm.nih.gov/pubmed/9326364, 415(1), 33-9.

[54] Carmignac, V, Svensson, M, Korner, Z, Elowsson, L, Matsumura, C, Gawlik, K. I, et al. Autophagy is increased in laminin a2 chain-deficient muscle and its inhibition improves muscle morphology in a mouse model of MDC1A. Hum Mol Genet. (2011). http://www.ncbi.nlm.nih.gov/pubmed/21920942, 20(24), 4891-902.

[55] Chamberlain, J. S, Metzger, J, Reyes, M, Townsend, D, \& Faulkner, J. A. Dystrophindeficient mdx mice display a reduced life span and are susceptible to spontaneous rhabdomyosarcoma. FASEB J. (2007). http://www.ncbi.nlm.nih.gov/pubmed/ 17360850, 21(9), 2195-204.

[56] Pauly, M, Daussin, F, Burelle, Y, Li, T, Godin, R, Fauconnier, J, et al. AMPK activation stimulates autophagy and ameliorates muscular dystrophy in the mdx mouse diaphragm. Am J Pathol. (2012). http://www.ncbi.nlm.nih.gov/pubmed/22683340, 181(2), 583-92.

[57] Doe, J. A, Wuebbles, R. D, Allred, E. T, Rooney, J. E, Elorza, M, \& Burkin, D. J. Transgenic overexpression of the a7 integrin reduces muscle pathology and improves viability in the $\mathrm{dy}(\mathrm{W})$ mouse model of merosin-deficient congenital muscular dystrophy type 1A. J Cell Sci. (2011). Pt 13):2287-97. http://www.ncbi.nlm.nih.gov/pubmed/ 21652631

[58] Sparks, S, Quijano-roy, S, Harper, A, Rutkowski, A, Gordon, E, Hoffman, E. P, et al. Congenital Muscular Dystrophy Overview. (1993). http://www.ncbi.nlm.nih.gov/ pubmed/20301468

[59] Irwin, W. A, Bergamin, N, Sabatelli, P, Reggiani, C, Megighian, A, Merlini, L, et al. Mitochondrial dysfunction and apoptosis in myopathic mice with collagen VI deficiency. Nature genetics. (2003). http://www.ncbi.nlm.nih.gov/pubmed/14625552, 35(4), 367-71. 
[60] Merlini, L, Angelin, A, Tiepolo, T, Braghetta, P, Sabatelli, P, Zamparelli, A, et al. Cyclosporin A corrects mitochondrial dysfunction and muscle apoptosis in patients with collagen VI myopathies. Proc Natl Acad Sci U S A. (2008). http:// www.ncbi.nlm.nih.gov/pubmed/18362356, 105(13), 5225-9.

[61] Palma, E, Tiepolo, T, Angelin, A, Sabatelli, P, Maraldi, N. M, Basso, E, et al. Genetic ablation of cyclophilin D rescues mitochondrial defects and prevents muscle apoptosis in collagen VI myopathic mice. Hum Mol Genet. (2009). http:// www.ncbi.nlm.nih.gov/pubmed/19293339, 18(11), 2024-31.

[62] Grumati, P, Coletto, L, Sandri, M, \& Bonaldo, P. Autophagy induction rescues muscular dystrophy. Autophagy. (2011). http://www.ncbi.nlm.nih.gov/pubmed/ 21543891, 7(4), 426-8.

[63] Oak, S. A, Zhou, Y. W, \& Jarrett, H. W. Skeletal muscle signaling pathway through the dystrophin glycoprotein complex and Rac1. J Biol Chem. (2003). http:// www.ncbi.nlm.nih.gov/pubmed/12885773, 278(41), 39287-95.

[64] Wei, Y, Pattingre, S, Sinha, S, Bassik, M, \& Levine, B. JNK1-mediated phosphorylation of Bcl-2 regulates starvation-induced autophagy. Molecular cell. (2008). http:// www.ncbi.nlm.nih.gov/pubmed/18570871, 30(6), 678-88.

[65] Yogev, O, Goldberg, R, Anzi, S, \& Shaulian, E. Jun proteins are starvation-regulated inhibitors of autophagy. Cancer Res. (2010). http://www.ncbi.nlm.nih.gov/pubmed/ 20197466, 70(6), 2318-27.

[66] Li, D. D, Wang, L. L, Deng, R, Tang, J, Shen, Y, Guo, J. F, et al. The pivotal role of cJun NH2-terminal kinase-mediated Beclin 1 expression during anticancer agents-induced autophagy in cancer cells. Oncogene. (2009). http://www.ncbi.nlm.nih.gov/ pubmed/19060920, 28(6), 886-98.

[67] Wei, Y, Sinha, S. C, \& Levine, B. Dual Role of JNK1-mediated phosphorylation of Bcl-2 in autophagy and apoptosis regulation. Autophagy. (2008). http:// www.ncbi.nlm.nih.gov/pubmed/18570871, 4(7), 949-51.

[68] Riol-blanco, L, Sanchez-sanchez, N, Torres, A, Tejedor, A, Narumiya, S, Corbi, A. L, et al. The chemokine receptor CCR7 activates in dendritic cells two signaling modules that independently regulate chemotaxis and migratory speed. J Immunol. (2005). http://www.ncbi.nlm.nih.gov/pubmed/15778365, 174(7), 4070-80.

[69] Djavaheri-mergny, M, Amelotti, M, Mathieu, J, Besancon, F, Bauvy, C, \& Codogno, P. Regulation of autophagy by NF-kB transcription factor and reactives oxygen species. Autophagy. (2007). http://www.ncbi.nlm.nih.gov/pubmed/17471012, 3(4), 390-2.

[70] Salminen, A, Hyttinen, J. M, Kauppinen, A, \& Kaarniranta, K. Context-Dependent Regulation of Autophagy by IKK-NF-kB Signaling: Impact on the Aging Process. Int J Cell Biol. (2012). http://www.ncbi.nlm.nih.gov/pubmed/22899934 
[71] Criollo, A, Senovilla, L, Authier, H, Maiuri, M. C, Morselli, E, Vitale, I, et al. The IKK complex contributes to the induction of autophagy. The EMBO journal. (2010). http:// www.ncbi.nlm.nih.gov/pubmed/19959994, 29(3), 619-31.

[72] Wu, Y. T, Tan, H. L, Huang, Q, Ong, C. N, \& Shen, H. M. Activation of the PI3K-AktmTOR signaling pathway promotes necrotic cell death via suppression of autophagy. Autophagy. (2009). http://www.ncbi.nlm.nih.gov/pubmed/19556857, 5(6), 824-34.

[73] Chen, Y, \& Klionsky, D. J. The regulation of autophagy- unanswered questions. J Cell Sci. (2011). Pt 2):161-70. http://www.ncbi.nlm.nih.gov/pubmed/21187343

[74] Mammucari, C, Schiaffino, S, \& Sandri, M. Downstream of Akt: FoxO3 and mTOR in the regulation of autophagy in skeletal muscle. Autophagy. (2008). http:// www.ncbi.nlm.nih.gov/pubmed/18367868, 4(4), 524-6.

[75] Jin, S. M, \& Youle, R. J. PINK1- and Parkin-mediated mitophagy at a glance. J Cell Sci. (2012). Pt 4):795-9. http://www.ncbi.nlm.nih.gov/pubmed/22448035

[76] Seo, A. Y, Joseph, A. M, Dutta, D, Hwang, J. C, Aris, J. P, \& Leeuwenburgh, C. New insights into the role of mitochondria in aging: mitochondrial dynamics and more. J Cell Sci. (2010). Pt 15):2533-42. http://www.ncbi.nlm.nih.gov/pubmed/20940129

[77] Guilak, F, Cohen, D. M, Estes, B. T, Gimble, J. M, Liedtke, W, \& Chen, C. S. Control of stem cell fate by physical interactions with the extracellular matrix. Cell Stem Cell. (2009). http://www.ncbi.nlm.nih.gov/pubmed/19570510, 5(1), 17-26.

[78] Lu, P, Weaver, V. M, \& Werb, Z. The extracellular matrix: a dynamic niche in cancer progression. J Cell Biol. (2012). http://www.ncbi.nlm.nih.gov/pubmed/22351925, 196(4), 395-406.

[79] Davies, K. E, \& Nowak, K. J. Molecular mechanisms of muscular dystrophies: old and new players. Nat Rev Mol Cell Biol. (2006). http://www.ncbi.nlm.nih.gov/ pubmed/16971897, 7(10), 762-73. 
